\title{
Pregnancies in women with diabetic nephropathy: long-term outcome for mother and child
}

\author{
R.Kimmerle ${ }^{1}$, R.-P.Zaß ${ }^{2}$, S. Cupisti ${ }^{1}$, T.Somville ${ }^{3}$, R. Bender ${ }^{1}$, B.Pawlowski ${ }^{4}$, M. Berger ${ }^{1}$ \\ ${ }^{1}$ Department of Metabolic Diseases and Nutrition, Heinrich-Heine University, Düsseldorf, Germany \\ ${ }^{2}$ Department of Pediatrics, Heinrich-Heine University, Düsseldorf, Germany \\ ${ }^{3}$ Department of Obstetrics and Gynecology, Heinrich-Heine University, Düsseldorf, Germany \\ ${ }^{4}$ Diabetes Research Institute, Düsseldorf, Germany
}

\begin{abstract}
Summary In order to improve the basis upon which to advise women with diabetic nephropathy about pregnancy, we studied the effect of diabetic nephropathy on the course of pregnancy, perinatal outcome, infant development and long-term outcome of the mothers. All pregnancies of women with diabetic nephropathy (defined as proteinuria $>400 \mathrm{mg} /$ day $(n=26)$, creatinine clearance $<80 \mathrm{ml} / \mathrm{min}$ and hypertension in the first trimester $(n=10))$ followed at our centre from 1982 to 1992 were identified ( 34 White class F and 2 White class T) and the women and their children re-examined in the spring 1993. From the first to the third trimester the percentage of women with proteinuria over $3 \mathrm{~g} /$ day increased from 14 to $53 \%$ and those treated with antihypertensive medication from 53 to $97 \%$. There were no intrauterine or perinatal deaths, but one child died suddenly 4 weeks postpartum. Of 36 newborns (gestational week at birth 36(3), birth weight 2384(834) g)), 11 were born before week 34 and 8 had respiratory distress syndrome. Renal function in the first trimester, diastolic blood pressure in the third trimester and an $\mathrm{HbA}_{1 \mathrm{c}}$ above normal were predictive of gestational age at delivery and low birth
\end{abstract}

weight (stepwise regression analysis). At follow-up of the children ( $n=35$, age $4.5(0.4-10)$ years) the majority $(n=27)$ were normally developed but seven had psychomotor retardation (four of them major). One child had a severe motor retardation due to a congenital anomaly. At follow up, 21 of the 29 mothers had preserved renal function (creatinine 1.3 (0.8-4.3) $\mathrm{mg} / \mathrm{dl}$ and 8 had developed end stage renal disease and required dialysis ( 2 of whom were White class T) within 3 (1-9) years postpartum. Of those, 4 women ( 3 White F and 1 White T) had died. Pregnancy did not seem to specifically accelerate the rate of decline of renal function. In women with diabetic nephropathy perinatal mortality can be prevented but perinatal and long-term infant morbidity remains elevated. Women with severely impaired renal function before pregnancy are at risk for serious morbidity when their children are still young. Improvement might be made if all women were to receive specialized care and counselling before, throughout and after pregnancy. [Diabetologia (1995) 38: 227-235].

Key words Diabetes mellitus, nephropathy, pregnancy.
Received: 27 April 1994 and in revised form: 24 August 1994

Corresponding author: Dr. R. Kimmerle, Department of Metabolic Diseases and Nutrition, WHO Collaborating Center for Diabetes, Heinrich-Heine University, Moorenstrasse 5, D-40225 Düsseldorf, Germany

Abbreviations: ESRD, end-stage renal disease; DSST, Denver developmental test.
Today the perinatal outcome of pregnancies of diabetic women without advanced diabetic complications is similar to that in non-diabetic women, if there is normoglycaemic control throughout pregnancy. Therefore, in women with well-controlled diabetes who do not have nephropathy or other advanced vascular complications their decision about whether or not to have children will largely be influenced by factors not associated with diabetes itself. In contrast, for women with diabetic nephropathy (who in most cases also have proliferative retinopa- 
Table 1. Clinical characteristics of women with diabetic nephropathy at the beginning of their pregnancies. For comparison the characteristics of 110 pregnancies of women of White

\begin{tabular}{lll}
\hline & With nephropathy & Without nephropathy \\
\hline Pregnancies/women $(n)$ & $40 / 33$ & $110 / 91$ \\
Age (years) & $29 \pm 5$ & $28 \pm 4$ \\
Duration of diabetes (years) & $20 \pm 5$ & $13 \pm 7^{\mathrm{c}}$ \\
Planned pregnancies $(n)$ & $12(30 \%)$ & $76(70 \%)^{\mathrm{d}}$ \\
Women with irregular menstrual periods $(n)$ & $26(65 \%)$ & $10(9 \%)^{\mathrm{d}}$ \\
Gestations/primipari $(n)$ & $1(1-14)^{\mathrm{a} / 26}(65 \%)$ & $1(1-3)^{\mathrm{b}} / 69(62 \%)$ \\
Initial visit to diabetic pregnancy clinic (gestational week) & $14 \pm 10$ & $11 \pm 6^{\mathrm{d}}$ \\
Elective abortion for medical reasons $(n)$ & $4(3 \mathrm{FR}, 1 \mathrm{~T})$ & 0 \\
Pregnancies $>12$ week of gestational/women $(\mathrm{n})$ & $36 / 29$ & $110 / 91$ \\
& $(31 \mathrm{FR}, 2 \mathrm{~F}, 1 \mathrm{HFR}, 2 \mathrm{~T})$ & $(36 \mathrm{~B}, 38 \mathrm{C}, 29 \mathrm{D}, 7 \mathrm{R})$ \\
History of hypertension $(n)$ & $22(61 \%)$ & $1(0.9 \%)$ \\
Antihypertensive medication before pregnancy $(n)$ & $19(53 \%)$ & 0 \\
Women legally blind $(n)$ & 3 & 0 \\
\hline
\end{tabular}

${ }^{a}$ Three women had a history of intrauterine deaths. ${ }^{b}$ One woman had a history of intrauterine death. ${ }^{c}$ Significantly different at $p=0.001$. ${ }^{\mathrm{d}}$ Significantly different at $p=0.02$

thy) concern about fetal and maternal complications remains a major issue [1-3]. Despite substantial improvements in perinatal survival with normoglycaemic control, treatment of hypertension and modern methods of assessment of fetal well-being and perinatal care, there is still a high rate of fetal morbidity largely due to prematurity [3].

There are indications that pregnancy itself does not accelerate the progression of diabetic nephropathy over and above that which might be expected in a comparable group of non-pregnant diabetic women, although controlled trials have not been performed [3-5]. However, with potential parents, the issue of the underlying prognosis of the woman's renal disease has to be addressed. Little recent information is available on the long-term effect of diabetic nephropathy before pregnancy on the mothers and their children $[6,7]$.

The purpose of the present study was to improve the basis upon which advice on pregnancy is given to women with diabetic nephropathy. While not specifically addressing issues of obstetric management, we attempted to elucidate factors before, during and after pregnancies associated with specific perinatal and long-term outcomes of mothers and their children. Therefore, we analysed all pregnancies in women with diabetic nephropathy seen at our centre from 1982 to 1992 and re-examined the women and their children in the spring of 1993.

\section{Subjects and methods}

Patient population. All pregnancies of White class $\mathrm{F}(n=37)$ or $\mathrm{T}(n=3)$ who were followed at the department of gynaecology and obstetrics of the Heinrich-Heine-University from 1982 to 1992 were identified. Pregnancies were included on the basis of persistent macroproteinuria ( $>400 \mathrm{mg} /$ day $(n=33)$ or $\geq 1+$ dipstick $(n=5)$ ) regardless of creatinine clearance and classes B, C, D and R cared for at our centre from 1987-1992 are shown. (Pregnancies with spontaneous abortions are not included)

- in the absence of macroproteinuria - on the basis of hypertension and a creatinine clearance less than $80 \mathrm{mg} / \mathrm{ml}(n=2)$ in the first trimester in the absence of urinary tract infection and other causes of renal disease. Pregnancies in which more than $400 \mathrm{mg} /$ day proteinuria developed only during the second half of pregnancy were not included. During such pregnancies $(n=40)$ the majority of women were additionally cared for at the diabetic pregnancy clinic of the department of metabolic diseases $(n=22)$ or at the diabetic pregnancy clinic of the Diabetic Research Institute in Düsseldorf $(n=12)$ and all but two women delivered babies in the department of obstetrics of our institution.

Information on early pregnancy and on the time before pregnancy was obtained through retrospective chart analysis, records from the women's private physician and from the prenatal "mother's pass", a booklet where findings (i.e., proteinuria, blood pressure) at each prenatal visit are documented and which remains with every woman cared for in Germany during and after pregnancies.

The women and their children were contacted in January 1993,4 months to 10 years after delivery or elective abortion and asked to attend our diabetic and pediatric clinic, for a follow-up examination.

The control group comprised 110 consecutive pregnancies of pregestational insulin-dependent diabetic women without nephropathy (White classes B, C, D and R) cared for jointly at the diabetic pregnancy clinics of the department of metabolism and nutrition and the department of obstetrics and gynaecology of the Heinrich-Heine-University from 1987-1992.

Care of women during pregnancy. Throughout pregnancy the women were seen every $2-3$ weeks at the obstetric and diabetic pregnancy clinics where $\mathrm{HbA}_{1 \mathrm{c}}$, blood pressure, weight and renal parameters were determined and insulin dose adjustments discussed. Retinal status was assessed by an ophthalmologist at least each trimester. During pregnancy the women used intensified insulin treatment with injection of regular insulin before each meal and NPH insulin in the morning and at bedtime $(n=34)$, or continuous subcutaneous insulin infusion $(n=6)$ with self-adaptations of insulin dose based upon frequent home blood glucose measurements 6-10 $\times /$ day) targeting blood glucose values between $3.3-5$ before and under $7.7 \mathrm{mmol} / \mathrm{l}$ after meals (Table 1$)$. Thirty $(72 \%)$ of the women had participated in a structured treatment and education pro- 
gramme independent of their pregnancy and were experienced in intensified insulin treatment. Such programmes also include information about family planning and pregnancy [8].

Assessment of the fetus included serial ultrasound imaging for fetal growth and anomalies. From week 26-28 the women were followed-up more frequently with regular fetal cardiotocogram assessment and admitted for intensified prenatal monitoring if indicated. Blood pressure management was based upon frequent home blood pressure measurements. If the blood pressure exceeded $140 / 90 \mathrm{mmHg}$ on several occasions despite bedrest, antihypertensive medication, which included methyldopa, hydralazin, atenolol (or metoprolol if serum creatinine exceeded $150 \mu \mathrm{mol} / \mathrm{l}$ ) was started or intensified alone or in combination. Diuretics were not used $[9,10]$.

Pre-eclampsia was diagnosed clinically and defined as acute worsening of hypertension ( $>15 \%$ of diastolic blood pressure) in the presence of proteinuria $>3 \mathrm{~g} /$ day and generalized oedema.

Timing and route of delivery were individualised on the basis of a balanced assessment of the maternal condition (renal function, hypertension, premature labour etc.), signs of fetal jeopardy (severe intrauterine growth retardation, positive contraction stress test or non-stress test).

Neonatal management. After delivery all children were examined and treated at the department of paediatrics. Infants whose birth weight exceeded the 90 th percentile for gestational age and sex were defined as large for gestational age and infants with a birth weight lower than the 10 th percentile were defined as small for gestational age [11]. Gestational age was determined by menstrual dates and modified by ultrasound examinations early in pregnancy and by physical examination of the newborn infant.

Postpregnancy follow-up. Follow-up examination of the women consisted of a history and physical, laboratory tests of renal function and a structured interview including social and reproductive issues. Blood pressure was measured according to World Health Organisation guidelines. Retinal status was assessed by an ophthalmologist. Information on the five women who had died was obtained from medical records, private physicians and family members.

The examination of the children was performed by a paediatrician and consisted of a neurological examination and for children under 6 years of age - the Denver developmental test (DSST). For the children older than 6 years of age the classification of psychomotor development was based upon school performance and the testing of representative psychomotor development functions such as speech development, motor co-ordination (i. e., finger nose, diadochokinesis), complex motor coordination (i. e. catching a ball), and static and dynamic balance (i.e., Romberg, standing on toes and on one leg and walking on heels and toes) [12]. Regular postnatal examinations as documented in the child's postnatal "Untersuchungsbuch" (examination booklet) by the German association of office based physicians (Kassenärztliche Vereinigung) were also used. In this booklet examinations at defined postnatal periods are outlined and the results documented by the private paediatrician. The standardized items tested are comparable to the items tested in the DSST. In 10 children a detailed written report about their health and development was obtained from their paediatrician or paediatric neurologist. Children with a normal neurological exam and a DSST index or neurodevelopmental functions within the normal range for their age were classified as "normal development". Children with mild abnormalities on neurological examination (i.e., abnormal movements, muscular hypotony, hyperreflexia) or who performed below the age standard for one function tested were classified as "mild neurodevelopmental retardation". Those who performed below the age range for several functions tested and who required special education or intensive remedial care were classified as "severe neurodevelopmental retardation".

Laboratory tests. $\mathrm{HbA}_{1 \mathrm{c}}$ was measured by HPLC (Biorad, Munich, Germany) and $\mathrm{HbA}_{1}$ by the column method (Boehringer-Mannheim, Mannheim, Germany) (normal range for nonpregnant control subjects $4.2-5.5 \%\left(\mathrm{HbA}_{1 \mathrm{c}}\right)$ and $4.0-8.0 \%$ $\left.\left(\mathrm{Hb}_{1}\right)\right)$. A glycated haemoglobin within the normal range for the respective method was defined as "good control", above the normal range as "poor control". Proteinuria, serum creatinine and creatinine clearance were measured by standard laboratory methods. Creatinine clearance was normalised to $1.73 \mathrm{~m}^{2}$ body surface.

\section{Statistical analysis}

Data are expressed as means (SD), as median and range or as frequencies. For comparison of means the parametric $t$-test and of proportions chi-square test was used. Bivariate associations were assessed by means of Pearsson's correlation coefficient or Spearman's rank analysis if data were not normally distributed. Stepwise linear regression and stepwise logistic regression was used to determine the combined effect of more than one variable on "gestational week at delivery" and "birth weight under $2000 \mathrm{~g}$ ", respectively [13]. Survival analysis, including the log rank test was performed to compare the probability of women with and without impaired renal function in early pregnancy to require dialysis during follow-up. Statistical analysis were done using SAS Technical Report, P200, SAS/STAT Software; Calis and logistic procedures, release 6.04 and SAS Technical Report P179. Additional SAS/ STAT. Procedures, Release 6.031980 (Lifetest; Cary, N.C., USA).

\section{Results}

Initial characteristics of women and their pregnancies. The clinical data of the women $(n=33)$ with diabetic nephropathy at the beginning of their pregnancies $(n=40)$ are shown in Table 1 . In four women (three White class $\mathrm{F}, 1 \mathrm{~T}$ ) pregnancy was electively terminated predominantly because of severely decreased renal function and uncontrolled hypertension before gestational week 12 . The bulk of this report concerns the 36 pregnancies of 29 women which went beyond gestational week 12 (23 women had one, five had two, and one woman had three pregnancies). During six of these pregnancies the women were only referred to the Düsseldorf centre after week 25 of pregnancy and previously had received non-specialized care. In two of these women (one of class T) pregnancy was diagnosed only in gestational weeks 20 and 26 , respectively. All but two women for whom pre-pregnancy information was not available had documented diabetic nephropathy before pregnancy (defined as proteinuria over $400 \mathrm{mg} /$ day or creatinine clear- 


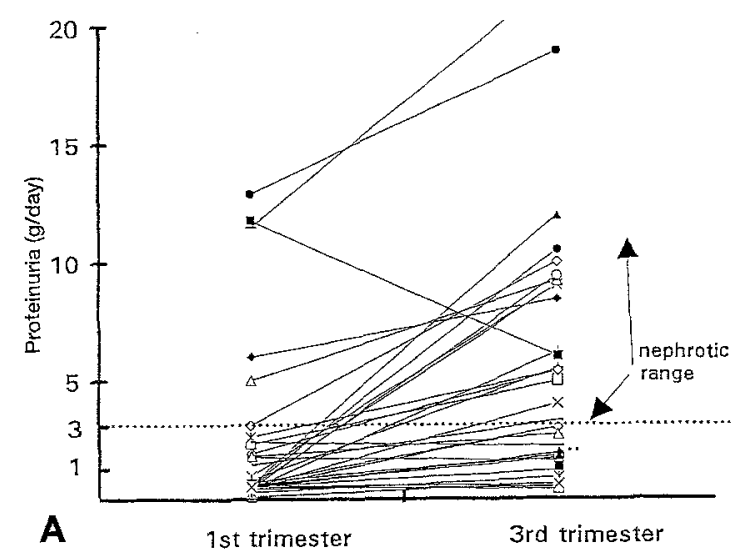

A

1st trimester

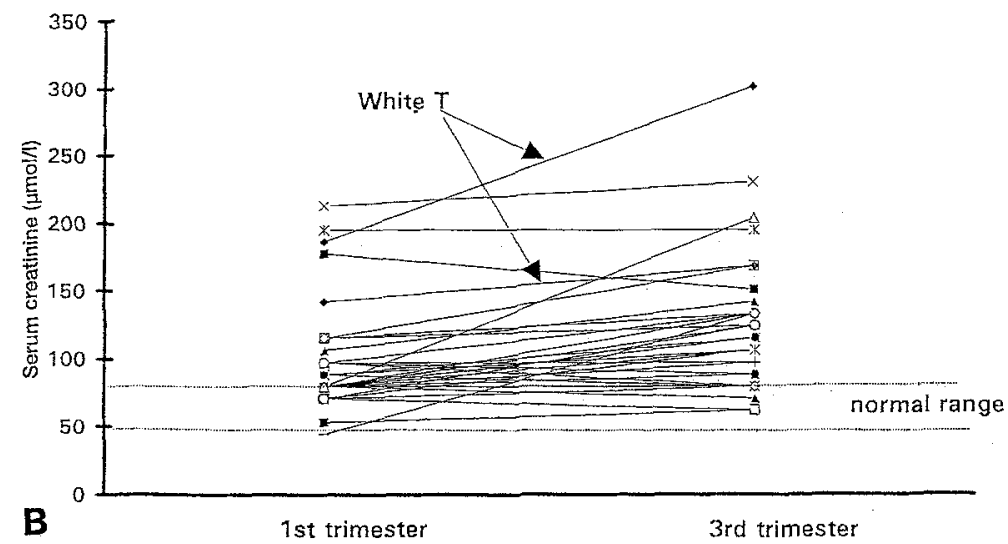

Fig. 1. (A, B) Proteinuria (A) and serum creatinine level (B) in the first and third trimester of pregnancy in women with diabetic nephropathy. In $\mathbf{B}$ the dotted lines indicate the range for serum creatinine in normal pregnancy

ance under $80 \mathrm{ml} / \mathrm{min}$ and hypertension). Renal function during the first trimester (defined as creatinine clearance $\geq 80 \mathrm{ml} / \mathrm{min}$ or - if serum creatinine only was available $(n=8)$ - as serum creatinine $<98 \mu \mathrm{mol} / 1)$, was preserved in 26 pregnancies, in $7 \mathrm{re}$ duced (serum creatinine $98-134 \mu \mathrm{mol} / 1$ and/or creatinine clearance $50-80 \mathrm{ml} / \mathrm{min}$ ) and in 3 severely reduced (serum creatinine $>134$ to $214 \mu \mathrm{mol} / \mathrm{l}$ and creatinine clearance 32,35 and $43 \mathrm{ml} / \mathrm{min}$ ).

The cardiac condition of the only woman of White class $\mathrm{H}$ (history of two myocardial infarctions) remained stable throughout pregnancy. All but two women (who had background retinopathy only) had been laser-treated for preproliferative or proliferative retinopathy before pregnancy. Three women were legally blind and three women had impaired vision (corrected vision $<40 \%$ ).

For comparison clinical data of 110 pregnancies of 91 pregestational insulin-dependent diabetic women without nephropathy cared for at our centre from 1987 to 1992 are shown (Table 1).

Maternal parameters during pregnancy. From the first to the third trimester proteinuria increased from 2.1 (1.1) to 5.0 (3.2) g/day (Fig. 1a). The percentage of women with proteinuria over $3 \mathrm{~g} /$ day increased from $5(14 \%)$ to $19(53 \%)$. Serum creatinine levels remained stable $( \pm 15 \%)$ in $22(61 \%)$ pregnancies and increased more than $15 \%$ in $14(39 \%)$ pregnancies (Fig. 1b). Creatinine clearance (available in early pregnancy for 28 pregnancies including all women with creatinine $>98 \mu \mathrm{mol} / \mathrm{l}$ ) decreased more than $10 \%$ in 14 and increased or remained within $\pm 10 \%$ in $14(50 \%)$ pregnancies from the first to the third trimester. In Table 2 maternal parameters during the third trimester are shown; all but one woman were treated with antihypertensive medication or increased doses of antihypertensives since blood pressure had increased above $140 / 90 \mathrm{mmHg}$. An intervariable correlation was found between urinary protein excretion and diastolic blood pressure in the third trimester $(r=0.58, p=0.0002)$. No significant correlation was found between serum creatinine level and diastolic blood pressure $(r=-0.08, p=0.07)$ and between proteinuria and serum creatinine in the third trimester $(r=0.12, p=0.5)$.

Seven $(19 \%)$ women developed a pre-eclampsialike syndrome as determined clinically with accelerating hypertension, generalised oedema, hypoalbuminaemia. Four women (two of White T) developed severe renal anaemia and had to be given a blood transfusion.

Glycaemic control in the second half of pregnancy as judged by the average monthly $\mathrm{HbA} / \mathrm{HbA}_{1 \mathrm{c}}$ from gestational week 26 to parturition was within the normal range in $81 \%$ and above the normal range in 7 $(19 \%)$ pregnancies. In $19(53 \%)$ of pregnancies retinal status deteriorated. Four of these women received laser treatment during the third trimester. Only one women had a permanent deterioration of her already impaired vision $(10 \%)$ as a result of a vitreous haemorrhage during pregnancy.

Thirty-one $(86 \%)$ of the infants were delivered by Caesarian section.

Perinatal outcome. There were no intrauterine or perinatal deaths. Gestational week at delivery for the group as a whole was 37 (29-40) and birth weight

Table 2. Maternal parameters of women with diabetic nephropathy in the third trimester of pregnancy

\begin{tabular}{ll}
\hline Number of pregnancies $(n)$ & 36 \\
\hline HbA $_{1 \mathrm{c}}$ normal range $(n)$ & $29(81 \%)$ \\
Systolic/diastolic blood pressure $(\mathrm{mm} \mathrm{Hg})$ & $152 \pm 16 / 90 \pm 10$ \\
Treatment with antihypertensives $(n)$ & $35(97 \%)$ \\
Proteinuria $>3$ g/day & $20(55 \%)$ \\
Pre-eclampsia-like syndrome & $7(19 \%)$ \\
Transient deterioration of retinopathy $(n)$ & $19(53 \%)$ \\
\hline
\end{tabular}




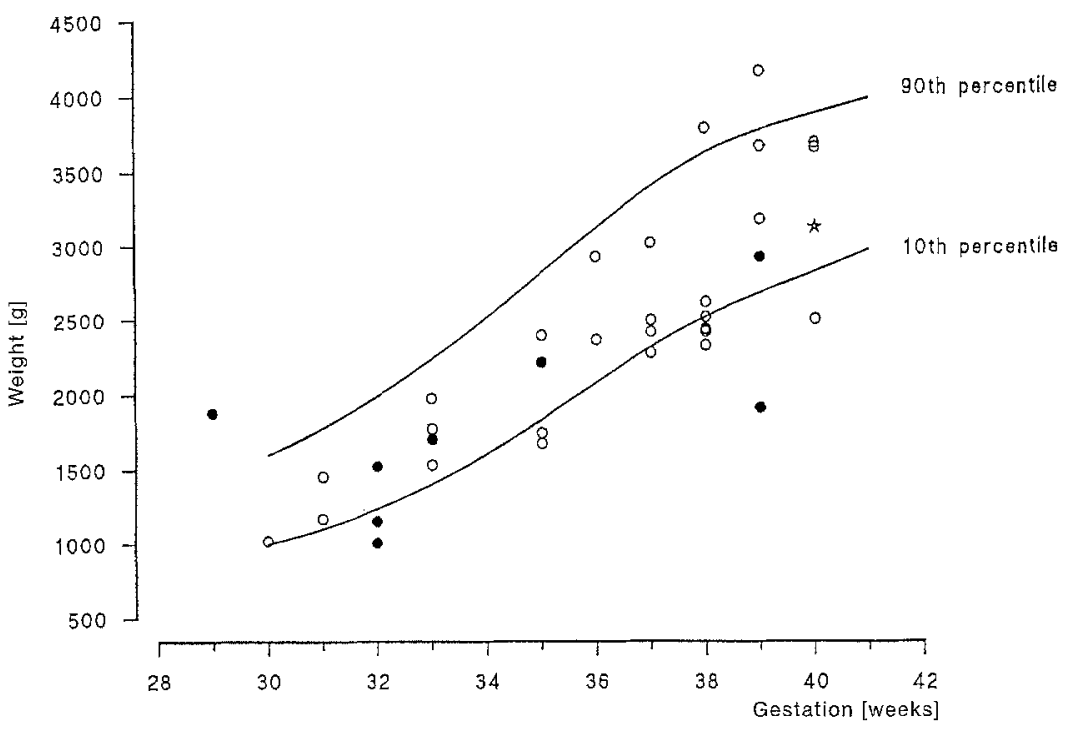

Fig. 2. Birth weight and gestational age at delivery of 36 newborns of mothers with diabetic nephropathy. Normally developed newborns (O); Newborns with neuro-developmental problems at follow-up ( ). * The newborn who died of SIDS. Lines indicate the 10 th and 90 th percentile for birth weight in the reference population [11]. Newborns below the 10th percentile are considered small for gestational age and above the 90th percentile large for gestational age

Table 3. Perinatal parameters in women with diabetic nephropathy according to their renal function at the beginning of their pregnancy. For comparison the perinatal outcome of 110 pregnancies of women of White classes B, C, D and R cared for at our centre from $1987-1992$ is shown

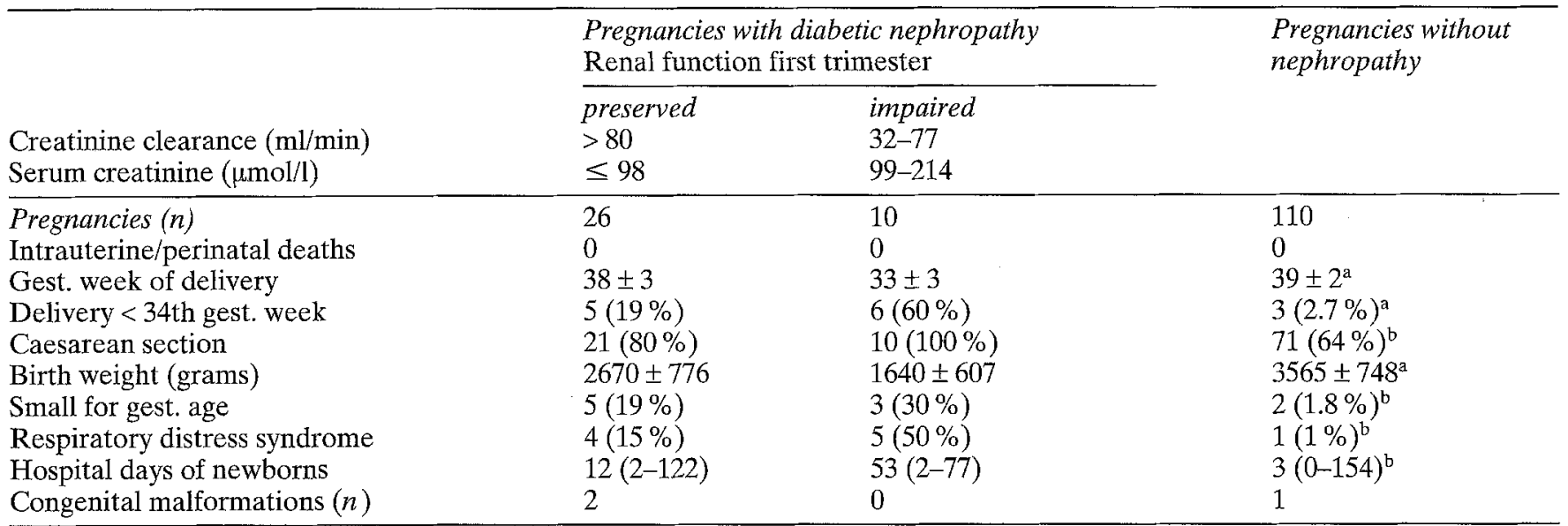

a Significantly different between each group $(p<0.05)$. ${ }^{b}$ Significantly different between the group without nephropathy vs the two groups with diabetic nephropathy $(p<0.05)$; not significantly different between the two groups with diabetic nephropathy

was $2365(1005-4130)$ g. There was a strong correlation between gestational age and birth weight $(r=0.87, p=0.0001)$ (Fig. 2). To determine which factors were predictive for gestational age at delivery a stepwise regression analysis was performed including log proteinuria, log serum creatinine and creatinine clearance and antihypertensive treatment in the first trimester; as well as systolic and diastolic blood pressure, glycaemic control and pre-eclampsia in the third trimester. In the final model diastolic blood pressure in the third trimester and creatinine clearance in the first trimester remained significantly predictive for gestational week at delivery $(p=0.01$ and $p=0.006$, respectively, $r^{2}=0.38$ ). To determine which factors were predictive for a "birth weight under $2000 \mathrm{~g}$ " a stepwise logistic regression was performed including log proteinuria, log serum creatinine and creatinine clearance and antihypertensive treatment during the first trimester; as well as systolic and diastolic blood pressure, glycaemic control and pre-eclampsia in the third trimester as potential predictive factors. In the final model diastolic blood pressure and poor glycaemic control in the third trimester remained as significant predictive factors $(p=0.01$ and $p=0.02$ ). The Hosmer-Lenneshow statistic indicated an adequate goodness-of-fit $(p=0.682)$.

In Table 3 perinatal parameters according to maternal renal function during the first trimester are shown. The nine newborns with respiratory distress syndrome were born prematurely ( 29 to 34 weeks) and received mechanical ventilation for 2-18 days. One of these children (gestational week of delivery 29), born before surfactant was available for the treatment of hyaline membrane syndrome, developed hypoxic brain damage and amaurosis premator- 
Table 4. Neurodevelopmental status at follow-up of 36 children of mothers whose pregnancy was complicated by diabetic nephropathy

\begin{tabular}{|c|c|c|}
\hline & $n$ & $\begin{array}{l}\text { Age at fol- } \\
\text { low-up } \\
\text { (years) }\end{array}$ \\
\hline Children & 36 & $0.5-11$ \\
\hline Deceased (SIDS at 5 weeks of age) & 1 & \\
\hline Normal neuro-developmental exam. & 27 & $0.5-11$ \\
\hline Mild developmental retardation & 3 & $0.4 ; 0.9 ; 4.2$ \\
\hline Severe developmental retardation & 2 & $5.8 ; 7$ \\
\hline Epilepsy, psychomotor retardation & 1 & 3 \\
\hline $\begin{array}{l}\text { Severe motor retardation } \\
\text { (congenital anomaly: arthrogryposis) }\end{array}$ & 1 & 5.1 \\
\hline $\begin{array}{l}\text { Tetraplegia, blindness, } \\
\text { severe psychomotor retardation }\end{array}$ & 1 & 6.5 \\
\hline
\end{tabular}

SIDS, Sudden infant death syndrome

Table 5. Status of 33 women with diabetic nephropathy $5(0.3-$ 9) years after pregnancy

\begin{tabular}{lll}
\hline & $\begin{array}{l}\text { Pregnancy } \\
\text { completed }\end{array}$ & $\begin{array}{l}\text { Elective } \\
\text { abortion }\end{array}$ \\
\hline $\begin{array}{l}\text { No. of women } \\
\begin{array}{l}\text { Women with normal serum } \\
\text { creatinine }(<98 \mu \mathrm{mol} / \mathrm{l})\end{array}\end{array}$ & 29 & 4 \\
$\begin{array}{l}\text { Women with serum creatinine } \\
\quad 98-178 \mu \mathrm{mol} / \mathrm{l}\end{array}$ & 10 & 1 \\
$\begin{array}{l}\text { Women with serum creatinine } \\
\quad>178 \mu \mathrm{mol} / \mathrm{l}\end{array}$ & 3 & 1 \\
$\begin{array}{l}\text { Women who had started dialysis } \\
\text { during follow-up } \\
\text { time between pregnancy and }\end{array}$ & $8(7 \mathrm{~F} ; 1 \mathrm{~T})$ & $2(1 \mathrm{~T})^{\mathrm{a}}$ \\
$\quad \begin{array}{l}\text { start of dialysis (years) } \\
\text { women deceased at follow-up }(n)\end{array}$ & $4(3 \mathrm{~F} ; 1 \mathrm{~T})$ & $1(\mathrm{~T})$ \\
$\begin{array}{l}\text { Women legally blind/visually } \\
\text { impaired }(n)\end{array}$ & $3 / 3$ & $0 / 3$ \\
\hline
\end{tabular}

a Of the 10 women who had started dialysis during follow-up eight had impaired renal function (serum creatinine $>98 \mu \mathrm{mol} / \mathrm{l})$ at the beginning of pregnancy.

$\mathrm{F}, \mathrm{T}$ : White classes $\mathrm{F}$ and $\mathrm{T}$

um. Two infants (maternal serum creatinine level in the first trimester $<98 \mu \mathrm{mol} / \mathrm{l}$ ) had a congenital malformation (arthrogryposis multiplex and atrial septal defect). The women had not attended specialised care before week 25 of pregnancy and were in poor glycaemic control in early pregnancy.

The perinatal parameters of 110 consecutive pregnancies of pregestational diabetic women without diabetic nephropathy are shown for comparison (Table 3).

Follow-up of children. One infant died suddenly at home 29 days postpartum (birth weight $3100 \mathrm{~g}$, gestational week at delivery 39). The neurological and developmental status of the remaining 35 children is shown in Table 4. Of the 31 children older than one year 4 had severe psychomotor and 1 severe motor retardation (congenital anomaly) requiring intensive remedial care and special education and 1 had minor psychomotor retardation (hyperactivity) requiring remedial care. Of the 4 children under 1 year of age at follow-up 2 had mildly retarded motor development and muscular hypotony (birth weight 1005 and $1740 \mathrm{~g}$ ) and 2 (birth weight 4020 and $2600 \mathrm{~g}$ ) were normally developed. Birth weight and gestational age at delivery of the children with and without neurodevelopmental problems is shown in Figure 2.

Follow-up of the women. The status of the 29 mothers and 4 women who underwent elective abortion is shown in Table 5. In the follow-up period 8 of the 29 $(28 \%)$ mothers had developed end-stage renal disease (ESRD) and had started dialysis (2 of the 2 women of White class T), 3 women within less than 1 year after delivery, 1 within 2 and 2 within 3 years and 1 within 4 and 9 years, respectively. Of the 8 mothers with ESRD 4 ( 1 of White class T) had died as a consequence of their renal disease, 3 within 3 and 1 within 4 years after delivery. Lifetable analysis revealed that the probability for requiring dialysis within the follow-up period was significantly greater for the 10 mothers with elevated serum creatinine ( $>98 \mu \mathrm{mol} / \mathrm{l}$ ) at the beginning of pregnancy than for those with normal creatinine $(p=0.03)$. The rate of fall of creatinine clearance could be estimated in 24 of the 27 mothers of White class F (excluding the women with White T). In the women who underwent more than one pregnancy during the study period the creatinine clearance from the most recent pregnancy was used. In three women data on creatinine clearance in early pregnancy were not available. The rate of fall of creatinine clearance was calculated as follows:

creatinine clearance at follow-up/start of dialysis minus creatinine clearance in early pregnancy divided by

months from beginning of pregnancy to follow-up/ start of dialysis.

(For start of dialysis a creatinine clearance of $10 \mathrm{ml} /$ min was assumed).

The rate of fall was $0.65(1.38) \mathrm{ml} / \mathrm{month}$. The rate of fall was greater in women who had started dialysis than in women not on dialysis at follow-up (2.43 (1.53) vs $0.18(0.9) \mathrm{ml} /$ month, $p<0.05)$. At follow up of the 21 women not on dialysis ( $66 \%$ of whom were taking antihypertensive medication, the blood pressure was $138(10) / 87$ (6) $\mathrm{mm} \mathrm{Hg}$, serum creatinine $116(71-383) \mu \mathrm{mol} / \mathrm{l}$, proteinuria 1607 (1027) $\mathrm{mg} /$ day and $\mathrm{HbA}_{1 \mathrm{c}} 8.5$ (1.6) \%. At follow-up the number of women legally blind $(n=3)$ and with severely im- 
paired vision $(n=3)$ had not increased compared to the beginning of pregnancy.

Social and reproductive issues at follow-up. At followup all women were living with their partners and all of them including the women on dialysis and those visually impaired performed the bulk of the child care and house work in their families. Two children were living in institutions for handicapped children. Eight women also worked full-time outside their homes. Six women had undergone sterilisation. Of the 21 women without ESRD, 9 had irregular menstrual periods and 11 intended to have another child.

\section{Discussion}

According to four previous reports, the fetal outcome of pregnancies for mothers with diabetic nephropathy has substantially improved since the 1970's compared to the 1960's when perinatal survival rates were below $70 \%[1-3,5-7]$. In our series of 36 pregnancies of 29 mothers there were no perinatal or intrauterine deaths but prematurity and associated perinatal morbidity was considerably higher than in pregnancies of diabetic women without nephropathy. For example, $31 \%$ of the infants of mothers with diabetic nephropathy had to be delivered before week 34 of gestation and $25 \%$ of newborns had respiratory distress syndrome in contrast to the nearabsence of these complications in 110 pregnancies of 91 insulin-dependent diabetic women without nephropathy followed at the same centre.

In this series the perinatal survival $(100 \%)$ was improved compared to two comparably large series reporting perinatal survival rates of 89 and $94 \%$, among 26 and 31 pregnancies complicated by diabetic nephropathy, respectively $[6,7]$. However, one of these series [6] included pregnancies from 1975 to 1978 and the treatment goals for glycaemic control since have shifted to lower values and might have improved outcome [5]. Respiratory distress syndrome in our series was related to premature delivery rather than to poor glycaemic control. In a recent series of Jovanovic and Jovanovic [5], which only included women with preconceptional normoglycaemic control only two of eight women with reduced creatinine clearance before pregnancy had to be delivered prematurely because of pre-eclampsia. However, this group seemed to have less severe impairment of renal function since it included only four women who had hypertension and proteinuria more than $1 \mathrm{~g} /$ day before pregnancy and since during the first trimester of pregnancy all but one woman had a creatinine clearance above $75 \mathrm{ml} / \mathrm{min}$. An elevated $\mathrm{HbA}_{1 \mathrm{c}}$ was also associated with a poorer perinatal outcome in our series. However, it remains doubtful whether normoglycaemia throughout pregnancy can prevent nearly all premature deliveries in women with reduced renal function as claimed by these authors. In our series 8 of the 11 women with very premature delivery were in good glycaemic control. Creatinine clearance during the first trimester and diastolic blood pressure during the third trimester were the variables most predictive for gestational age at delivery.

In our series $53 \%$ of the women had received antihypertensive drug treatment before their pregnancies and during the third trimester in all but one pregnancy. The women were treated with antihypertensive medication because of diastolic blood pressures increasing above $90 \mathrm{~mm} \mathrm{Hg}$. Therefore, diastolic blood pressure values exceeding $100 \mathrm{~mm} \mathrm{Hg}$ for prolonged periods were rare. In view of the fact that diastolic blood pressure during the third trimester was still predictive of gestational age at delivery it seems indicated to treat elevations of diastolic blood pressure above $90 \mathrm{~mm} \mathrm{Hg}$. In accordance with previous observations proteinuria increased between the first to the third trimester and was in the nephrotic range in $53 \%$ of pregnancies $[6,7]$. Proteinuria during the third trimester was correlated to increasing diastolic blood pressure. Of the women already treated with antihypertensive medication (predominantly betablockers) at the beginning of their pregnancy a similar percentage had a massive proteinuria ( $>3 \mathrm{~g} /$ day) in the third trimester compared to those not treated. This is remarkable because proteinuria in early pregnancy tended to be higher, although not significantly, in the women treated early in pregnancy. The underlying poorer renal function in most of these women might have played a role; i.e., with less functioning glomeruli less protein could be lost [14]. However, it is conceivable that antihypertensive medication early in pregnancy reduced the pregnancy-specific, hormone-induced increase in renal hyperfiltration and thus prevented a massive loss of protein by already damaged glomeruli $[15,16]$.

For non-diabetic pregnancies complicated by chronic, non-proteinuric, mild to moderate, hypertension it has been reported that fetal and maternal outcomes were satisfactory and it has also been suggested that diabetic pregnancies should not to start drug treatment before diastolic blood pressure exceeded $105 \mathrm{~mm} \mathrm{Hg}[5-7,9,10]$. However, it appears that in pregnant women with diabetic nephropathy blood pressure treatment should start at lower values also in order to prevent a massive loss of protein and hypoalbuminaemia. Hypoalbuminaemia causing a further decrease in intravascular volume might contribute to decreased uteroplacental blood flow which is believed to be one of the causes of pre-eclampsia [17].

In our series there were two infants with major congenital malformations both born to women with preserved renal function but poor glycaemic control in early pregnancy. Although not statistically com- 
parable because of the small numbers, this compares unfavourably with one malformation that occurred in the control group of 110 pregnancies of pregestational diabetic women without diabetic nephropathy. It is now well-accepted that if glycaemic control is good in early pregnancy advanced microangiopathic complications are not specifically associated with an increased risk for congenital malformations [5, 18]. At follow-up, the majority of the children including 8 of the 11 children born before week 34 were normally developed but there was a high rate of children $(25 \%)$ with psychomotor and developmental problems. The problems were varied and difficult to classify and might, at least in those with milder retardation, improve or resolve with advancing age [19]. Although the number of children was small and methods of ascertainment and classification not comparable, the percentage of children with psychomotor problems, and their severity appeared to be clearly greater than that reported for children of unselected groups of diabetic mothers encompassing all White classes [20-23]. In the two most recent of these series it was found that children of insulin-dependent diabetic mothers followed at age 4-5 years had an essentially normal cognitive development albeit a slightly increased risk for - mostly minor - motor developmental problems in comparison to children born to non-diabetic mothers [22, 23].

Kitzmiller et al. [6] did find two children with psychomotor retardation (both related to their congenital neurological anomalies) among 24 surviving infants of mothers with diabetic nephropathy examined at age 8 to 35 months. Reece et al. [7] reported essentially normal development in 27 children (on average 3 years old at follow-up) of mothers with diabetic nephropathy. However, these series were not quite comparable since there were intrauterine and perinatal deaths, and the children were younger. Moreover, in Reece's series [7] information on the children was only obtained through the infant's private physicians and was not standardized. Thus, on the basis of the available data no conclusions about a specific influence of maternal diabetic nephropathy on long-term infant outcome can be made, other than that there might be an increased risk for developmental problems because of a high rate of low birth weight infants. In our series six of eight children with psychomotor problems had a birth weight under $2500 \mathrm{~g}$ and were born before gestational week 37 . Bloch-Petersen et al. [23, 24] also found that low birth weight and prematurity were associated with an increased risk of poor performance at age 4 5 years when examining 90 children of diabetic mothers in Denmark. Moreover, large series of children weighing $<2000$ g or less at birth, born to non-diabetic mothers, found significantly more, albeit mostly minor, developmental problems at school age than in infants with higher birth weight $[19,20,25]$.
An important finding was the outcome of the mothers, i.e. 6 of 27 women (excluding the 2 women of White class T) progressing to ESRD within 1 to 9 years after delivery. Kitzmiller et al. [6] observed 3 of 23 diabetic women progressing to ESRD within 2 years after delivery and Reece et al. [7] 6 of 27 (average follow up 3 years). In these series, which had a shorter follow-up, none of the women had died during follow-up in contrast to our series where 3 of 27 mothers (excluding the 2 women of White class $T$ ) had died within 5 years after pregnancy. Most authors conclude that there is no influence of pregnancy on the course of diabetic nephropathy, although controlled trials have not been performed [3-5]. Jovanovic and Jovanovic [5] found in their series of 8 patients that the pregnancy-induced increase in proteinuria and change of renal function had returned to pregestational levels within the first year after pregnancy.

For the majority of women of White class $F$ in our series, there is circumstantial evidence that pregnancy did not specifically accelerate the course of diabetic nephropathy although we cannot make definite conclusions since complete data on renal function before pregnancy and between delivery and follow-up were not available in all women. The average rate of fall in creatinine clearance of $0.65 \mathrm{ml} / \mathrm{min}$ per month was slightly above the range which has been observed in prospective studies on the course of diabetic nephropathy employing meticulous antihypertensive control $(0.1-0.45 \mathrm{ml} / \mathrm{min} / \mathrm{month})[16]$. However, in analogy to such studies, the rate of decline of renal function varied considerably among the women observed here and tended to be steep in those with already reduced creatinine clearance, and minimal or non-existent in the majority of women with normal creatinine clearance before pregnancy. At follow-up, the women without ESRD had satisfactory blood pressure control. It is known that through effective blood pressure management deterioration of renal function can be considerably slowed, particularly in non-smokers, even in patients who have advanced diabetic renal disease [16]. This supports the notion that also during pregnancy the treatment goal for blood pressure should be at lower values than are usually recommended for non-diabetic pregnant women with chronic hypertension $[9,10]$. Moreover, after pregnancy close medical follow-up by specialists in diabetic nephropathy must continue.

Both of the women with renal transplants (creatinine clearance 35 and $32 \mathrm{ml} / \mathrm{min}$ in early pregnancy) required dialysis 1 and 2 years after delivery because of progressive transplant rejection, which had started in one of these women before, and in one during, pregnancy. One of these women died 3 years after delivery. Both their children were born very prematurely and were small for gestational age and had psychomotor retardation at follow-up (age 1 year and 
5 years old). One woman who had a renal transplant and had followed the advice to have an abortion died 4 years after her pregnancy. Thus, it appears that pregnancy should be discouraged in diabetic women who have reduced renal transplant function [26].

A major problem in the group of women with nephropathy, which might have influenced outcome, was the high rate of unplanned pregnancies. In these women planning of pregnancy and preconceptional care is not only important for the improvement of glycaemic control but also for rigorous treatment of hypertension and retinal problems $[3,5]$. Significantly more women with nephropathy had unplanned pregnancies and attended specialized care late during pregnancy than in the group of diabetic women without nephropathy. This might be partly due to the high rate of women with irregular menstrual periods. Many of these women had considered themselves infertile and did not use birth control. On the other hand, factors such as poor acceptance of their diabetes and poor compliance with medical advice might be implicated. Although, we did not initially design the study to examine these issues, such women seemed over-represented among the women with diabetic nephropathy.

In summary, with normoglycaemic control, tight blood pressure management and contemporary methods of fetal surveillance and perinatal care, perinatal mortality but not all perinatal morbidity can be prevented in pregnancies of women with diabetic nephropathy. Some children may have psychomotor problems at later age. Women with severely impaired renal function before pregnancy are at risk for serious morbidity when their children are still young. Improvements might be achieved if all women receive specialized care and counselling before, during and after pregnancy.

\section{References}

1. Dicker D, Feldberg D, Peleg D, Karp M, Goldman JA (1986) Pregnancy complicated by diabetic nephropathy. J Perinat Med 14: 299-307

2. Hare JW, White P (1977) Pregnancy in diabetic complicated by vascular diseases. Diabetes 26: 953-955

3. Nesler ChL, Sinclair SH, Schwartz SS, Gabbe SG (1985) Diabetic nephropathy in pregnancy. Clin Obstet Gyn 288: 528-535

4. Hayslett JP, Reece EA (1987) Effect of diabetic nephropathy on pregnancy. Am J Kidney Dis 9: 344-349

5. Jovanovic R, Jovanovic L (1984) Obstetric management when normoglycemia is maintained in diabetic pregnant women with vascular compromise. Am J Obstet Gynecol 149: 617-623

6. Kitzmüller JL, Brown ER, Phillippe M et al. (1981) Diabetic nephropathy and perinatal outcome. Am J Obstet Gynecol 141: 741-752

7. Reece EA, Coustan DR, Hayslett JP et al. (1988) Diabetic nephropathy: pregnancy performance and fetomaternal outcome. Am J Obstet Gynecol 159: 56-66
8. Mühlhauser I, Bruckner I, Berger M et al. (1987) Evaluation of an intensified insulin treatment and teaching program as routine management of type I (insulin-dependent) diabetes. The Bucharest-Düsseldorf Study. Diabetologia 30: $681-690$

9. Cunningham GF, Lindheimer MD (1992) Hypertension in pregnancy. In: Desforges JF (ed) Current concepts. NEJM 326: $927-932$

10. Redman CWG (1982) Controlled trials of treatment of hypertension during pregnancy. Obstet Gynecol Surv 37: $523-530$

11. Largo P, Walli S (1980) Intrauterines und extrauterines Wachstum (Kinderkliniken Zürich und Winterthur. Helv Paed Acta 35: 419-425

12. Towen BCI (1979) Examination of the child with minor neurological dysfunction. In: Clinics in developmental medicine 71. Heinemann Medical Books, London

13. SAS/Stat guide for personal computers. 6th edition, 1987, SAS Institute Inc., Cary, NC, USA

14. Mogensen CE, Christensen CE, Christensen WJ et al. (1981) Renal function handling in normal hypertensive and diabetic men. Contrib Nephrol 24: 139-145

15. Katz AI, Davison JM, Hayslett JP, Sinson E, Lindheimer MD (1980) Pregnancy in women with kidney disease. Kidney Int 18: 192-206

16. Parving $\mathrm{HH}$, Andersen AR, Smidt UM, Hommel E, Mathiesen ER, Svendsen PA (1987) Effect of antihypertensive treatment on kidney function in diabetic nephropathy. BMJ 294: 1443-1447

17. Soffronoff EC, Kaufmann BM, Connaughton JFF (1977) Intravascular volume determinations and fetal outcome in hypertensive diseases of pregnancy. Am J Obstet Gynecol 127: 4-9

18. Hanson U, Persson B, Thunell S (1990) Relationship between hemoglobin $\mathrm{A}_{1 \mathrm{c}}$ in early type I (insulin-dependent) diabetic pregnancy and the occurrence of spontaneous abortion and fetal malformation in Sweden. Diabetologia 33: 100-104

19. McCormick MC, Brooks-Gunn J, Workman-Daneils K, Turner J, Peckham GJ (1992) The health and developmental status of very low-birth-weight children at school age. JAMA 267: 2204-2208

20. Michelsson K, Lindahl E, Parre M, Helenius M (1984) Nine-year follow-up of infants weighing $1500 \mathrm{~g}$ or less at birth. Acta Paediatr Scand 73: 835-841

21. Cummins M, Norrish A (1980) Follow-up of children of diabetic mothers. Arch Dis Child 55: 259-264

22. Persson B, Gentz J (1984) Follow-up of children of insulindependent and gestational diabetic mothers. Acta Paediatr Scand 73: 349-358

23. Bloch-Petersen M (1989) Status at 4-5 years in 90 children of insulin-dependent diabetic mothers. In: Stowers, Sutherland HW (eds) Carbohydrate metabolism in pregnancy and the newborn. Springer, N. Y. pp 354-361

24. Michelsson K, Noronen M (1983) Neurological, psychological and articulatory impairment in five-year-old children with birthweight of $2000 \mathrm{~g}$ or less. Eur J Pediatr 141: 96-100

25. Peterson MB, Pedersen SA, Greisen G, Pedersen JF, Molsted-Pedersen L (1984) Early growth delay in diabetic pregnancy: relation to psychomotor development at age 4 . BMJ 283: 269-271

26. Ogburn PL, Kitzmiller JL, Hare JW (1986) Pregnancy following transplantation in class $\mathrm{T}$ diabetes mellitus. JAMA 255: 911-915 